\title{
Cosmology with the Square Kilometer Array by SKA-Japan
}

Daisuke Yamauchi*

Kanagawa University

E-mail: yamauchi@jindai.jp

In the past several decades, the standard cosmological model has been established and its parameters have been measured to a high precision, while there are still many of the fundamental questions in cosmology; such as the physics in the very early Universe, the origin of the cosmic acceleration and the nature of the dark matter. The future world's largest radio telescope, Square Kilometre Array (SKA), will be able to open the new frontier of cosmology and will be one of the most powerful tools for cosmology in the next decade. The cosmological surveys conducted by the SKA would have the potential not only to answer these fundamental questions but also deliver the precision cosmology. In this article the cosmology science led by the SKA-Japan Consortium (SKA-JP) Cosmology Science Working Group is discussed.

11th International Workshop Dark Side of the Universe 2015

14-18 December 2015

Yukawa Institute for Theoretical Physics, Kyoto University Japan

${ }^{*}$ Speaker. 


\section{Introduction}

In the past several decades, by accurately measuring the statistical properties of the cosmic microwave background (CMB) anisotropies, large-scale structures, supernovae and gravitational lensing, a simple standard cosmological model has been estabilished and its parameters have been measured to a high precision. Our Universe is well described by a spatially flat, $\Lambda$ cold dark matter (CDM) model with nearly scale-invariant, adiabatic and Gaussian primordial fluctuations. But the high-precision measurements have revealed that only 5\% in the enegy budget of the Universe seems to be made of the ordinary atomic matter [1]. In other words, the remaining $95 \%$ seems to be composed of exotic dark components such as dark matter and dark energy.

Even though the standard cosmological model has been estabilished, there are still many fundamental questions in cosmology. The remaining issues in cosmology would be as follow: What type of the inflationary model is actually realized in the very early Universe? What is the mechanism of generating primordial fluctuations? What is the dark matter? What is the physical origin of the cosmic acceleration, namely the dark energy or the modification of gravity theory?

Although the CMB has been one of powerful observational tools for cosmology in the past decades, the next step towards a precision cosmology will require extra information. The future world's largest radio telescope, Square Kilometre Array (SKA) ${ }^{1}$, will provide the threedimensional information by measuring the large scale strucuture, and will be one of the most powerful tools for cosmology and play an important role in addresing these issues.

\section{Cosmology with the SKA by SKA-Japan consortium}

The SKA has the potential to deliver the very precise cosmological measurements through the HI galaxy survey [2, 3, 4], HI intensity mapping survey [5 6] and radio continuum survey [7] . We expect that the cosmological survey conducted by the SKA will yield tranformational science across a wide range of cosmology. In this section, we summarize the SKA-oriented cosmological scientific challenges in which the cosmologists in Japanese research community, called SKA-Japan consortium (SKA-JP) cosmology science working group, have a deep interest. We have wide expertise in both theoretical/observational cosmology and are particularly interested in the studies of the very early stage of the Universe, the observational tests of the general relativity on cosmological scales and the particle physics such as the neutrinos and the beyond-standard-model physics. While observing the small-scale is of great interest from various perspectives for both cosmology and particle physics, it is hard to provide the accurate predictions on such scales, mainly because density perturbations grow significantly and enter within highly nonlinear regime. Moreover, the feedback of the baryonic physics is poorly understood and involves large uncertainties. In order to obtain the unprecedented constraints on the parameters for the fundamental physics, we need to focus on the observation on the specific redshifts and scales. This section is a brief overview of the science that SKA can achieve and our community is currently playing an important role.

\footnotetext{
${ }^{1}$ See http://www.skatelescope.org
} 


\subsection{Ultra-large scale cosmology with multitracer technique}

One of the possible ways to overcome the issues discussed above is to focus on the observations on the ultra-large cosmological scales. Although it is hard to access ultra-large scales, theoretical prediction on such scales can be very precise, because in superhorizon scale, the growth of the density perturbation fully remains within the linear regime and the effects of the baryonic physics feedback due to the astrophysical processes are sufficiently suppressed. However, the clustering analysis at large scales are limited due to cosmic-variance, due to the lack of enough independent measurements. A promising way to access the ultra-large scales is to reduce the cosmic-variance with so-called multitracer technique [8 9], in which the availability of multiple tracers with the different biases allows significant improvements in the statistical error.

\subsubsection{Multitracer technique and synergy with Euclid survey}

Among various cosmological parameters characterizing the primordial Universe, we focus on the primordial non-Gaussianity. Many members of Japanese cosmology community have been extensively working on the inflationary model-building and the theoretical studies of the mechanism of generating the primordial non-Gaussianity during the inflationary epoch. Although primordial non-Gaussianity has primarily been constrained from measurements of CMB so far, the resultant constraint has almost saturated to the observational limit predicted by ideal observations. Large scale halo/galaxy distributions provide distinct information on the primordial non-Gaussianity. Luminous sources such as galaxies must be most obvious tracers of the underlying dark matter distributions with a bias. For the standard Gaussian initial conditions, the halo bias is often assumed to be linear, deterministic, and scale-independent. However, it was found that the non-Gaussianity in primordial fluctuations effectively introduces a scale-dependent clustering of galaxies on large scales. The modification appears in a biased tracer of the underlying matter distribution. The nonGaussian correction to the large-scale Gaussian bias of a biased tracer, induced by the local-type non-Gaussianity $f_{\mathrm{NL}}$, is given in [10 11]. The effect of the local type primordial non-Gaussianity on the halo bias is prominent on large scales and at high redshifts. Thus, in the large-scale limit, the non-Gaussian correction become dominant, and the enhancement of clustering amplitude becomes significant. With the help of this property, the constraint on primordial non-Gaussianity has been obtained (see e.g., [12]13).

D. Yamauchi and K. Takahashi, who are members of SKA-JP, have discussed the potential power of the multitracer technique with future galaxy surveys in [14. We focus on the SKA radio continuum survey [7] . In our treatment, the specific population of galaxies is considered to match the extragalactic simulation performed in [15], assuming that the sensitivity limit of $1(0.1) \mu \mathrm{Jy}$ and applying a $5 \sigma$ detection for SKA1(2). In this case, they correspond to the detection of $5 \times 10^{8}$ and $10^{9}$ galaxies, respectively. Moreover, to split the galaxy samples into the subsamples by the inferred halo mass and redshift, five radio galaxy types such as star-forming galaxies, radio quite quasars, radio-loud AGN and starbursts are considered to infer the halo mass. We actually have shown that the SKA radio continuum survey can constrain up to the accuracy of $\sigma\left(f_{\mathrm{NL}}\right) \approx 0.7$ for SKA2, and $\sigma\left(f_{\mathrm{NL}}\right) \approx 1.6$ for SKA1 (see also [16]). Even when the GR horizon-scale effects are taken into account, it is shown that $\sigma\left(f_{\mathrm{NL}}\right) \lesssim 1$ can be achieved [17] 18]. 


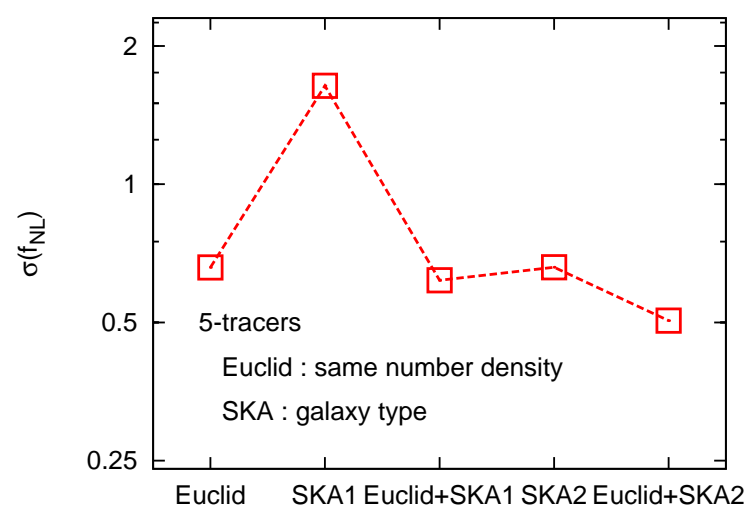

Figure 1: The expected marginalized constraints on $f_{\mathrm{NL}}$ for each survey and combinations. Constraints $\sigma\left(f_{\mathrm{NL}}\right) \lesssim 1.8$ and 0.7 can be obtained with a single survey of SKA1 and Euclid, respectively. The combination of SKA and Euclid can provide a stronger constraint $\sigma\left(f_{\mathrm{NL}}\right) \lesssim 0.65$ for SKA1+Euclid and an unprecedented constraint $\sigma\left(f_{\mathrm{NL}}\right) \lesssim 0.5$ for SKA2+Euclid. [14

At the time when the SKA is operational, there will be additional survey from a number of ground-based telescopes and space missions. Euclid is expected to be launced in 2020 and will perform imaging and spectroscopic surveys in optical and infrared bands. While the number of galaxies $\left(\sim 10^{8}\right)$ and covered sky area $\left(\sim 15,000 \mathrm{deg}^{2}\right)$ for Euclid are relatively smaller than these for SKA, we consider Euclid photometric galaxy survey as a future representative survey that will be conducted on comparable time scales. Fig. 1 shows that constraints $\sigma\left(f_{\mathrm{NL}}\right) \lesssim 1.8$ and $\lesssim 0.7$ can be obtained with a single survey of SKA1 and Euclid, respectively. The combination of SKA and Euclid can provide a stronger constraint $\sigma\left(f_{\mathrm{NL}}\right) \lesssim 0.65$ for SKA $1+$ Euclid and an unprecedented constraint $\sigma\left(f_{\mathrm{NL}}\right) \lesssim 0.5$ for SKA2+Euclid, implying that most inflationary models with even small primordial non-Gaussianity can be excluded by the combination of future galaxy surveys (see also [19, 20]).

\subsubsection{Higher-order primordial non-Gaussianity and their consistency relation}

Constraining various properties of primordial non-Gaussianity is expected to provide the oppotunity to break the degeneracy of inflationary models, because its amplitude, shape, and scaledependence encode much information of the primordial Universe. In other words, different nonlinear parameters are linked to distinctive meachnisms that can generate such non-Gaussianity during/after the inflationary epoch.

One of the major theoretical discoveries from the studies of inflationary models is that all inflationary models predict the presence of the consistency relation between nonlinear parameters. For the simplest scenarios, if there is the nonvanishing local-form bispectrum, the trispectrum must necessarily exist with $\tau_{\mathrm{NL}}=\left((6 / 5) f_{\mathrm{NL}}\right)^{2}$, where $\tau_{\mathrm{NL}}$ denotes the local-type nonlinear parameter charactering the amplitude of the primordial trispectrum [21, 22]. Even in a general situation, one can show that there is a universal relation, $\tau_{\mathrm{NL}} \geq\left(\frac{6}{5} f_{\mathrm{NL}}\right)^{2}$, which is often refereed to the Suyama- 

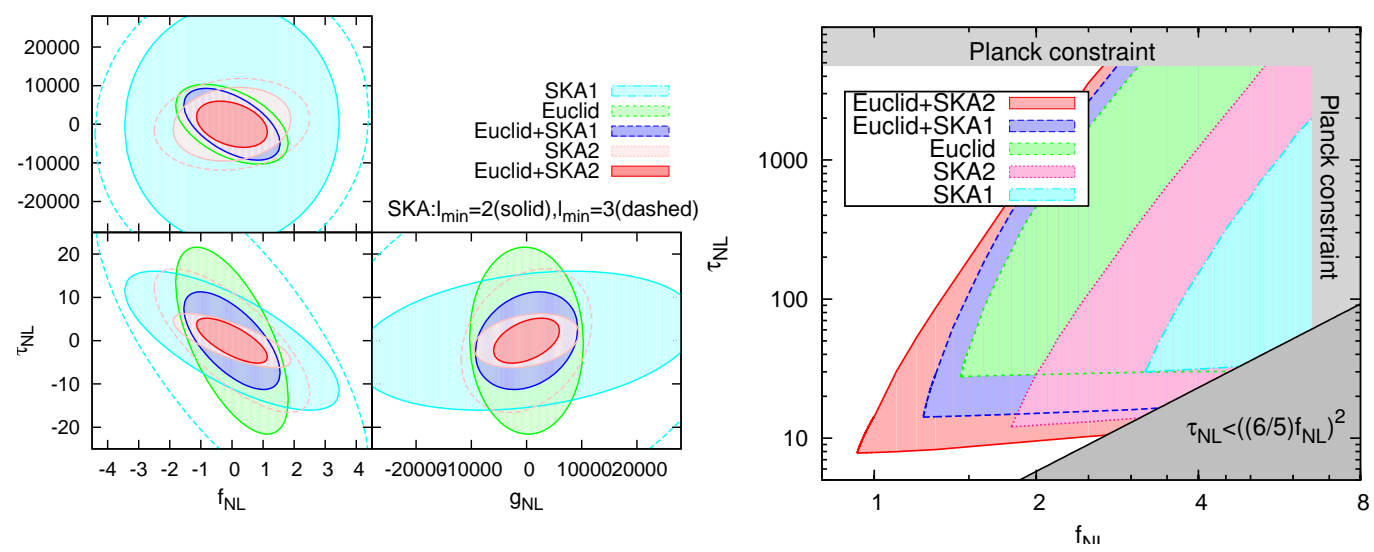

Figure 2: Left:Forecast $1 \sigma$ marginalized contours in $\left(f_{\mathrm{NL}}, \tau_{\mathrm{NL}}\right),\left(g_{\mathrm{NL}}, \tau_{\mathrm{NL}}\right)$ and $\left(f_{\mathrm{NL}}, g_{\mathrm{NL}}\right)$ planes with the vanishing fiducial values of the nonlinear parameters. To see the dependence on the minimal multipole we also plot the results for SKA with $\ell_{\mathrm{min}}^{\mathrm{SKA}}=3$ in dashed line. Right:Parameter space to confirm the consistency relation, $f_{\mathrm{NL}} / \sigma\left(f_{\mathrm{NL}}\right)$ and $\tau_{\mathrm{NL}} / \sigma\left(\tau_{\mathrm{NL}}\right)$. For comparison, the inconsitent region and the constraints from Planck are also shown in gray color. When $f_{\mathrm{NL}} \lesssim 1.5$, the confirmation of the consistency inequality is rather challensing for a single survey. Combining SKA2 and Euclid drasically improves the constraint to reach the confirmation even for $f_{\mathrm{NL}} \approx 0.9$ and $\tau_{\mathrm{NL}} \approx 8$. [32]

Yamaguchi inequality [23 24]. Primordial trispectrum is also usually characterized by another nonlinear parameter, $g_{\mathrm{NL}}$, which corresponds to the strength of the intrinsic cubic nonlinearities of primordial fluctuations. A detection of the higher-order primordial non-Gaussianity and the confirmation of the inequality from large-scale structure measurements would indicate the presence of more complicated dynamics, e.g., multi-field inflationary models (see [25, 26, 27 for curvaton model), in the primordial Universe. Thus, the detection and confirmation should be the target in future experiments (see [28]). Furthermore, we could rule out not only the simplest single-field but also the multifield inflation models if we find the breaking of the consistency inequality.

When $\tau_{\mathrm{NL}}>\left((6 / 5) f_{\mathrm{NL}}\right)^{2}$, the halo density contrast is not fully correlated to the matter linear density field even in the absence of the shot noise. Namely, the simple bias relation $\delta_{\mathrm{h}}=b_{\mathrm{h}} \delta$ should be modified due to the stochasticity. This provides the unique oppotunity of large-scale scale-dependent stochastic bias as a probe of the primordial Universe associated with complicated dynamics 29, 30, 31].

D. Yamauchi and K. Takahashi have discussed the required survey level needed to probe the higher-order primordial non-Gaussianity such as $\tau_{\mathrm{NL}}, g_{\mathrm{NL}}$ and test the consistency relation with future galaxy surveys [32] (see also [33 ). In order to see the impact of the higher-order primordial non-Gaussianity on the parameter estimation, the $1 \sigma$ marginalized contour obtained through the Fisher analysis is shown in Fig. 2. Assuming the specific distribution of galaxies introduced in the previous subsubsection, as for $\tau_{\mathrm{NL}}$, the SKA radio continuum survey can reach $\sigma\left(\tau_{\mathrm{NL}}\right) \approx 4(11)$ for the SKA2(1), which is an improvement by a factor of 100 (25) compared with the Planck constraint, suggesting that the deep survey and large sky coverage provided by SKA are advantageous to constrain $\tau_{\mathrm{NL}}$, and the information even from the early stage of SKA is quite essential to break 
the degeneracy between these nonlinear parameters. The constraint on $g_{\mathrm{NL}}$ from SKA is also several tens of times severer than that obtained from Planck. Concentrating on $f_{\mathrm{NL}}$ and $\tau_{\mathrm{NL}}$, the dependence of our forecast on the choice of the fiducial values is studied in Fig. 2, where we show the region in which both $f_{\mathrm{NL}}$ and $\tau_{\mathrm{NL}}$ are detected at $1 \sigma$ significance. When $f_{\mathrm{NL}}$ is small, say $\lesssim 1.5$, the confirmation at $\gtrsim 1 \sigma$ level is rather challenging for a single survey, even when we apply the multitracer technique. However, the combination of SKA and Euclid drastically improves the constraints and can detect the consistency relation in the wide parameter region. These results shows that the SKA radio continuum survey combined with the future optical galaxy surveys such as Euclid offers an unique oppotunity to probe the detail dynamics in the primoridial Universe.

\subsubsection{Halo/galaxy bispectrum}

The primordial bispectrum and the higher-order moments measure the fundamental interactions and nonlinear processes occuring during inflation. Not only the amplitude but also the shape of the bispectrum encodes much physical information of the primordial Universe. Different shape can be also linked to different mechanism for generating non-Gaussian fluctuations. It would be very interesting to try further constraining various types of primordial non-Gaussianity by precise large-scale structure measurements, which are expected to contain much more information than CMB. It is, however, shown that the scale-dependent clustering due to non-local type models is weaker than the local-type [34, 35, 36, 37, implying that the measurements of the scale-dependent clustering in the galaxy power spectrum is irrelevant. The effect of the scale-dependent bias sourced by primordial non-Gaussianity appears not only in the galaxy power spectrum but also in the galaxy bispectrum [38]. Indeed, the amplitude of the galaxy bispectrum sourced by the equilateral-type primordial bispectrum and trispectrum, which do not lead to an enhancement in the galaxy power spectrum, is shown to be enhanced on large scales [39 40]. Although the late-time nonlinear gravitational evolution effectively gives the nonvanishing contributions to the observed galaxy bispectrum, the different scale-dependence on large scales is expected to give stringent constraints on the non-local type primordial non-Gaussianity. Hence our community will try to constrain the non-local type primordial non-Gaussianity with the help of the multitracer technique.

\subsection{Exploring the dark Universe with $21-\mathrm{cm}$ survey}

In recent years, observations of HI signals (i.e. $21 \mathrm{~cm}$ line) emitted at the Epoch of Reionization (EoR) have attracted much attention because the observations will open a new window to the early phases of cosmological structure formations. Since there are a lot of hydrogen gas in the IGM at the very high redshift Universe $(6 \gtrsim z)$, by observing the redshifted $21 \mathrm{~cm}$ line with SKA-low we can survey such very past epochs, and not only study how the Universe was ionized but also obtain information about density fluctuations of matters.

The $21 \mathrm{~cm}$ line results from hyperfine splitting of the $1 \mathrm{~S}$ ground state due to an interaction of magnetic moments of proton and electron, and we can define the spin temperature $T_{S}$ through a ratio between number densities of hydrogen atom in the $1 \mathrm{~S}$ triplet and $1 \mathrm{~S}$ singlet states, $n_{1} / n_{0} \equiv$ $\left(g_{1} / g_{0}\right) \exp \left(-T_{\star} / T_{S}\right)$, where $T_{\star} \equiv h c / k_{B} \lambda_{21}=0.068 \mathrm{~K}$ with $\lambda_{21}(\simeq 21 \mathrm{~cm})$ being the wave length of the $21 \mathrm{~cm}$ line at a rest frame, and $g_{1} / g_{0}=3$ is the ratio of spin degeneracy factors of the two states. A difference between the observed $21 \mathrm{~cm}$ line brightness temperature at redshift $z$ and the 
$\mathrm{CMB}$ temperature $T_{\mathrm{CMB}}$ is given by

$$
\begin{aligned}
& \Delta T_{b}(r, z) \simeq 26.8 x_{\mathrm{HI}}\left(1+\delta_{b}\right)\left(\frac{\Omega_{b} h^{2}}{0.023}\right)\left(\frac{0.15}{\Omega_{m} h^{2}} \frac{1+z}{10}\right)^{1 / 2} \\
& \quad \times\left(\frac{1-Y_{p}}{1-0.25}\right)\left(\frac{T_{S}-T_{\mathrm{CMB}}}{T_{S}}\right)\left(\frac{H(z) /(1+z)}{d v_{\|} / d r_{\|}}\right) \mathrm{mK},
\end{aligned}
$$

where $r$ is the comoving coordinate, $x_{\mathrm{HI}}$ is the neutral fraction of hydrogen, $Y_{p}$ is the primordial ${ }^{4} \mathrm{He}$ mass fraction, $\delta_{b}$ is the hydrogen density fluctuation, and $d v_{\|} / d r_{\|}$is the gradient of the proper velocity along the line of sight due to both the Hubble expansion and the peculiar velocity. Through the contribution of $\delta_{b}$ in the differential brightness temperature of $21 \mathrm{~cm}$ line, we can obtain the information of matter density fluctuations.

The growth of the density fluctuations depends on cosmological parameters, and we can use the $21 \mathrm{~cm}$ line surveys for constraining them like CMB observations or galaxy surveys. Since the redshifted frequency of $21 \mathrm{~cm}$ line corresponds to each past era, the $21 \mathrm{~cm}$ line surveys enable us to observe very wide redshift ranges ( $21 \mathrm{~cm}$ tomography). Therefore, we can obtain a large number of Fourier samples and information about time evolution of density fluctuations. Additionally, in such high redshift eras, non-linear growth of density fluctuations is less effective in comparison with that in later epochs, and we can treat their time evolution with linear theory up to relatively small scales. For this reason, in the $21 \mathrm{~cm}$ line surveys, theoretical uncertainties due to the growth of density fluctuations are much smaller than those in galaxy surveys. These features are advantages of the $21 \mathrm{~cm}$ line surveys.

In a work studied by K. Kohri, Y. Oyama, T. Sekiguchi, T. Takahashi, who are members of SKA-JP [41], they found that by using combinations of CMB and $21 \mathrm{~cm}$ line we can precisely measure the primordial curvature perturbations, which are generated by inflation. In the previous section, we take account of higher correlation functions, but in this work they focus on the two point correlation function (i.e. power spectrum) of the primordial curvature perturbations. The two point and higher correlation functions are complementary to each other, and both correlation functions are important in order to discriminate inflation models. In most of previous works about discriminating the inflation models, mainly focused parameters were spectral index $n_{s}$ of the power spectrum and the dependence of $n_{s}$ on the wave number (i.e. spectral running $\alpha_{s}$ ). However, these parameters are not insufficient to distinguish the inflation models in some cases, and we need higher order wave number dependence of the power spectrum, e.g. the wave number dependence of $\alpha_{s}$ (i.e. running of running $\beta_{s}$ ). Though generally the running of running is very small and it is difficult to measure its value, in that work, they found that we can strongly improve its constraints by combining CMB experiments with precise $21 \mathrm{~cm}$ line surveys such as SKA (see Fig. 3).

Also, we can study elementary particle physics by the $21 \mathrm{~cm}$ line surveys. For example, K. Kohri and Y. Oyama have investigated how well we can constrain properties of neutrino; the sum of the neutrino masses $\Sigma m_{v}$, the effective number of neutrino species $N_{v}$, and the hierarchy of neutrino masses 444 45]. Neutrinos are elementary particles with neutral electric charge, and interact only through the weak interaction with other particles.

Since the discoveries of neutrino masses by Super-Kamiokande through neutrino oscillation experiments in 1998, the standard model of particle physics has been forced to change to theoretically include the neutrino masses. So far, only mass-squared differences of neutrinos have been 

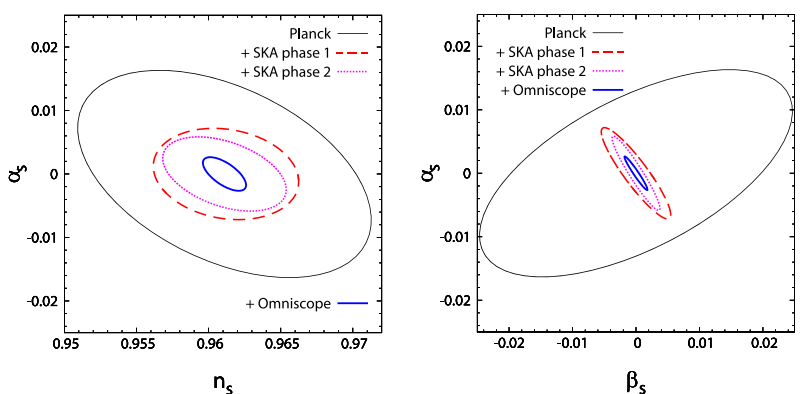

$\mathrm{n}_{\mathrm{s}}$
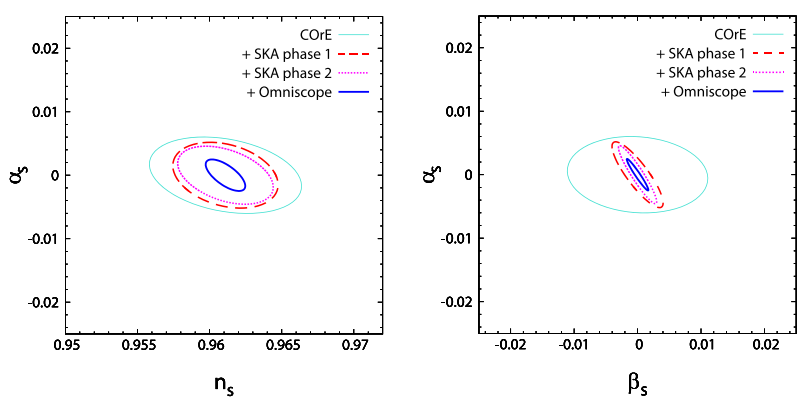

Figure 3: Contours of 95\% C.L. forecasts by combinations of CMB (Planck (upper) and COrE (lower)) and SKA (phase 1 and phase 2) in the $n_{s}-\alpha_{s}$ (left) and $\alpha_{s}-\beta_{s}$ (right) planes. We set the observed redshift range to be $6.75<z<10.05$. SKA can strongly constraint on $n_{s}, \alpha_{s}$ and $\beta_{s}$ in comparison with cases of CMB only. For comparison, we also plot forecasts by Omniscope, which is a future square-kilometer collecting area array optimized for $21 \mathrm{~cm}$ line survey [42]43]. [41]

measured by neutrino oscillation experiments. However, the absolute values of neutrino masses and hierarchical structure of them (normal or inverted) have not been measured yet although their information is indispensable to build new particle physics models. On the other hand, such nonzero neutrino masses affect cosmology significantly because relativistic neutrinos erase the gravitational potential up to the free streaming scale. Additionally, the effective number of neutrino species contributes to an extra radiation component and affects the expansion rate of the Universe. Besides, the hierarchical structure of neutrino masses affects both the free-streaming scales and the expansion rate. Therefore, we can obtain constraints of the neutrino masses, the effective number of neutrino species and the hierarchy of neutrino masses from cosmological observations.

By using SKA1, we can strongly constrain on the sum of the neutrino masses $\Sigma m_{v}$ and the effective number of neutrino species $N_{v}$ (see Fig. (4). In future, by using SKA2, there is a possibility of distinguishing the hierarchy of neutrino masses unless the mass structure is degenerated (see Fig. 5). Moreover, they found that we can also obtain strong constraints of the lepton number asymmetry of the Universe [46], which may become a relatively large value in some models of elementary particle physics. From Fig. 6 adding $21 \mathrm{~cm}$ line surveys by SKA to CMB observations, we see that constraints of the lepton number asymmetry are greatly improved in comparison with those of CMB only.

Thus far, physicists have investigated properties of elementary particles by ground-based experiments. However, by using $21 \mathrm{~cm}$ line surveys, we can measure detailed behaviors of matter fluctuations, and information of them enables us to clarify properties of elementary particles. This 


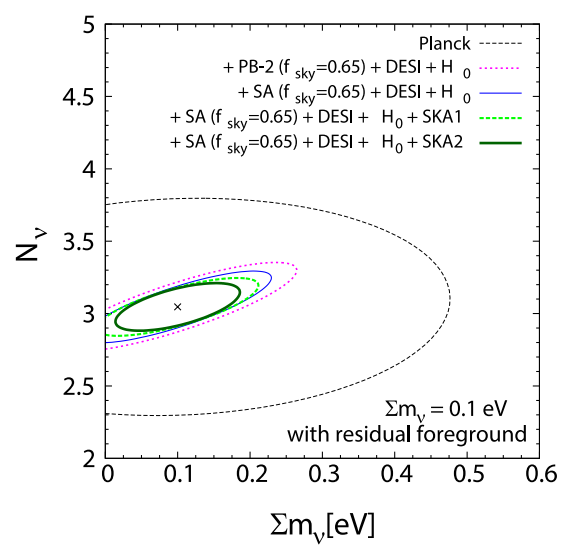

Figure 4: Contours of $95 \%$ C.L. forecasts in the $\Sigma m_{v}-N_{v}$ plane by combinations of CMB (Planck, PoLARBEAR-2 (PB-2), Simons Array (SA)) and SKA (Phase 1 and Phase 2). Fiducial values of neutrino parameters, $N_{v}$ and $\Sigma m_{v}$, are taken to be $N_{v}=3.046$ and $\Sigma m_{v}=0.1 \mathrm{eV}$. We set the observed redshift range to be $6.75<z<10.05$. POLARBEAR-2 and Simons Array are ground-based precise CMB polarization observations, and DESI is a future baryon acoustic oscillation (BAO) observation. [45]
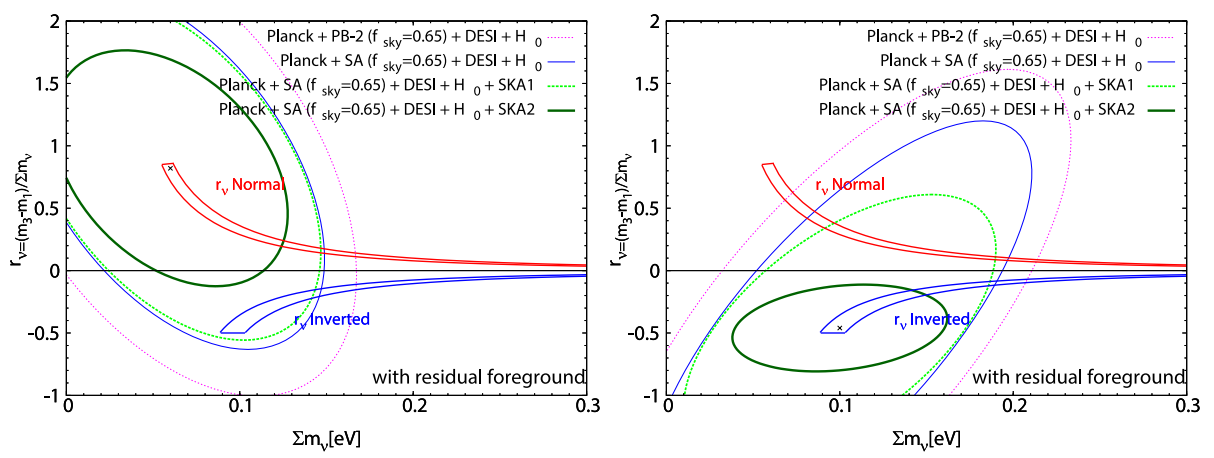

Figure 5: Contours of 95\% C.L. forecasts in the $\Sigma m_{v}-r_{v}$ plane by combinations of CMB (Planck, PoLARBEAR-2 (PB-2), Simons Array (SA)) and SKA (Phase 1 and Phase 2), where $r_{v} \equiv\left(m_{3}-m_{1}\right) / \Sigma m_{v}$. We assume that an observed redshift range is $6.75<z<10.05$. Allowed parameters on $r_{v}$ by neutrino oscillation experiments are also plotted as two bands for the inverted and the normal hierarchies, respectively (the name of each hierarchy is written in the close vicinity of each band). [45]

point is specialties of the works stated above. Therefore, in our research plans, we aspire for obtaining strong constraints of cosmological parameters by observing the high redshift Universe with the use of the $21 \mathrm{~cm}$ line surveys by SKA. In particular, the members of SKA-JP are world leading scientists in the research about particle cosmology by using $21 \mathrm{~cm}$ line surveys.

In the previous section, we focus attention on biased density fluctuations at super horizon scales. On the one hand, in this section, we take particular note of the behavior of density fluctuations at relatively small scales, and both research are complementary to each other. Also, for the 21 $\mathrm{cm}$ line surveys, we plan to investigate how precisely $21 \mathrm{~cm}$ line observations can constrain models of the dark energy and the primordial non-Gaussianity, which have been the focus of the study in the previous section. Moreover, we also pay attention to surveys of HI intensity mapping. Thus far, 

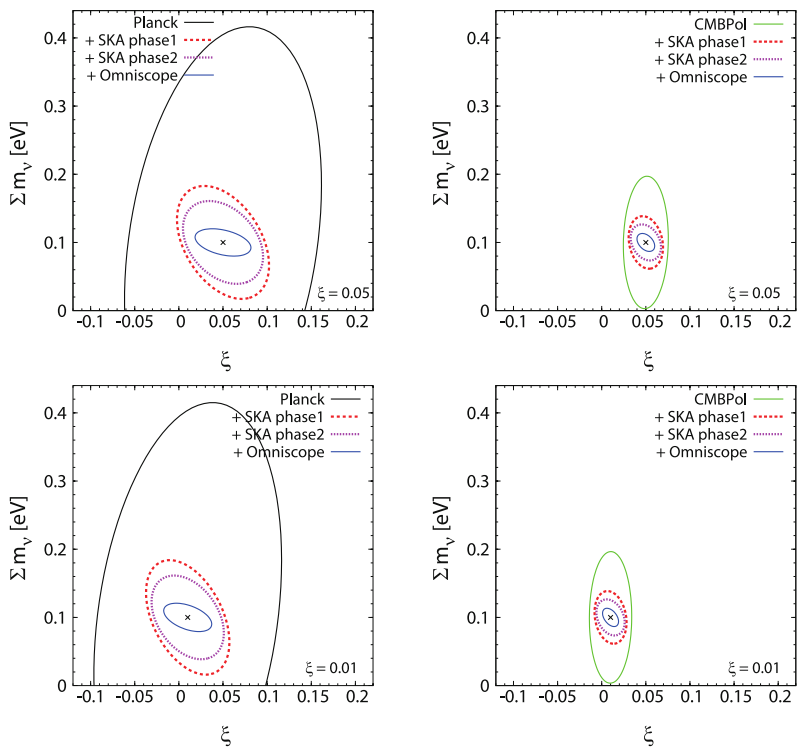

Figure 6: Contours of $95 \%$ C.L. forecasts in the $\Sigma m_{v}-\xi$ plane, where $\xi \equiv \mu_{v} / T_{v}$ represents the lepton asymmetry of the Universe, $\mu_{v}$ is the neutrino chemical potential, and $T_{v}$ is the temperature of relic neutrinos. As CMB data, the Planck and CMBPol surveys are adopted in the left and right panels, respectively. In order from top to bottom, the fiducial values of $\xi$ are set to be 0.05 and 0.01 . We assume that an observed redshift range is $6.75<z<10.05$. For comparison, we also plot forecasts by Omniscope. [46]

research about the HI intensity mapping with the motivation to study elementary particle physics are not considered so much, and we expect that this method by using the intensity mapping will develop hereafter.

Also, signals of $21 \mathrm{~cm}$ line strongly depend on astrophysical processes in the epoch of reionization. However, we do not know sufficiently about the reionization history yet. In addition, foregrounds of $21 \mathrm{~cm}$ line surveys are very strong radiation and removal of them is one of the most important subjects. Members of the reionization group of SKA-JP particularly focus on the astrophysical processes in the epoch of reionization and the removal of foregrounds. Therefore, by working with the members of the reionization group, we can obtain more accurate theoretical models of $21 \mathrm{~cm}$ line signals and methods for the removal of foregrounds. The models and methods enables us to reduce theoretical uncertainties in the $21 \mathrm{~cm}$ line surveys.

\subsection{Cosmic shear measurements with SKA}

Precision weak lensing measurement in cosmology is a key observable for revealing the latetime evolution of density perturbations. The weak lensing mesurement conducted by the SKA radio continuum survey 47 will probe the galaxy population to higher redshift that the lensing signal becomes larger. The shape (or shear) of galaxies modified by gravitational lensing can be characterized by even and odd-parity modes (E- and B-mode, respectively) 448 49]. The symmetric argument implies that the density perturbations at linear-order produce only the E-mode of the cosmic shear field (see e.g. [50]). Hence, non-vanishing B-mode signals on large angular scales immediately imply the presence of non-scalar perturbations. Although the amplitude of these sig- 
nals is small, it is known that there are a variety of generating mechanism. Possible subdominant components in the Universe, such as active vector modes generated by topological defects [51], primordial gravitational waves [52] and secondary vector/tensor modes generated by density perturbations [53, 54], rather than first-order density perturbations, can induce the B-mode shear. A search for those tiny signals from the B-mode shear is a key to explore subdomiant components of the Universe.

In [53] we found that the total B-mode shear signals cannot exceed the expected noise level even for the weak lensing measurement conducted by the SKA2, while the signal induced by the second-order vector mode turns out to dominate on almost all scales. On the other hand, the weak lensing measurement in the HI IM survey is expected to substantially improve the signal-to-noise ratio and provide the observable signals, because the angular power spectrum of the brightness temperatures do not have diffusion scale unlike $\mathrm{CMB}$ and measures the fluctuations at different epochs. We leave this for future investigations.

\subsection{Synergy between SKA and CMB experiments: primordial gravitational-waves}

Measurements of the odd-parity pattern (B-modes) in the CMB polarization on large angular scales have been considered as the best avenue to probe the primordial gravitational-waves as a smoking gun of the cosmic inflation [55]. Still, there is no evidence for the presence of the primordial gravitational-waves, and the amplitude of the primordial gravitational-waves relative to the primordial density fluctuations, usually referred to as the tensor-to-scalar ratio $r$, is constrained as $r<0.07$ (95\% C.L.) from the Joint analysis of BICEP2, Keck Array and Planck Collaborations [56 57]. The detection of the gravitational-wave (primary) B-modes is one of the main targets in ongoing and future $\mathrm{CMB}$ experiments.

On large angular scales, B-modes from Galactic foreground emission are expected to dominate over the primary B-modes. Multiple studies (e.g., [59, 60, 61]) have been devoted for foreground cleaning techniques (see [62] for a resent review). 61] showed that the foreground contamination would be possible to be subtracted enough to detect the primordial B-modes at $r \sim 0.001$. However, even if the foreground contamination is successfully mitigated, there is still a significant contamination from the gravitational lensing effect on the CMB polarization caused by the gravitational potential of the large scale structure [63]. Precise estimate and subtraction of the lensing B-modes, usually referred to as delensing [64 65, 66, will be required in ongoing and future CMB experiments such as BICEP/Keck Array series [58] and LiteBIRD [67].

The lensing B-modes can be estimated from measurements of the CMB-lensing mass fields or using Cosmic Infrared Background (CIB) intensity map. The CMB-lensing mass fields have been precisely reconstructed from CMB polarization experiments 68, 69, 70, 71] (see [72] for a recent review), and the lensing B-modes estimated from the CMB-lensing mass fields have been detected with high significance very recently [71]. Detections of the lensing B-modes from the CIB observations have been also reported in several CMB experiments [74 69, 71]. These lensing Bmodes are, however, measured at scales where the primary B-modes have negligible contribution. The delensing with these lensing B-modes therefore does not improve the sensitivity to the primary B-modes.

The estimate of the lensing B-modes would be also possible using any CMB-lensing mass tracers such as halos/galaxies at high redshifts which generate the most part of the gravitational 


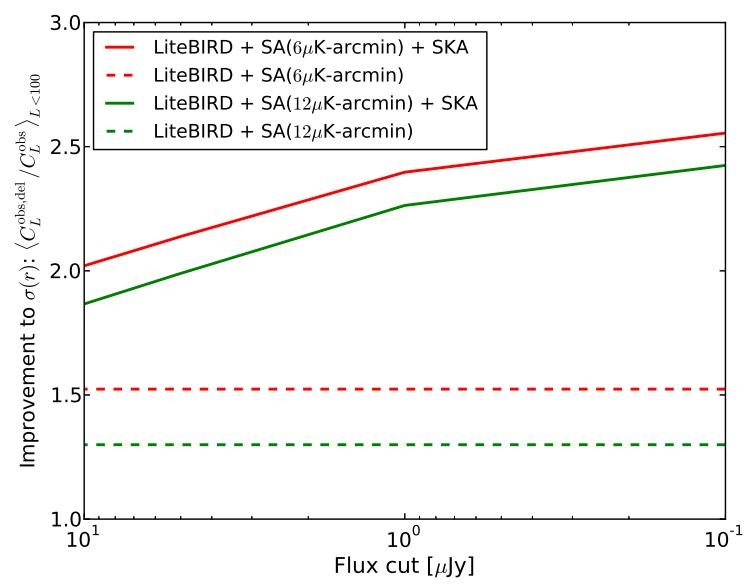

Figure 7: Delensing improvement to constraints on the tensor-to-scalar ratio as a function of the flux cut of the SKA radio continuum survey. The solid lines show the delensing improvement by combining the LiteBIRD, Simons Array (SA) and SKA, while the horizontal dashed lines show the case with CMB experiments alone. The red (green) lines show the case if the polarization sensitivity of the Simons Array becomes $6 \mu \mathrm{K}$-arcmin $(12 \mu \mathrm{K}$-arcmin). (see [78])

potential of the CMB lensing [75, 76, 77]. The SKA radio continuum survey [7] will provide a two-dimensional intensity map of the synchrotron radiations from halos/galaxies integrated along the line of sight. These sources would be located at high redshifts $(z \sim 2)$. Since the CMB lensing is mostly generated by inhomogeneities of the mass distribution at such redshifts, fluctuations in the intensity map measured from the radio continuum survey would effectively trace the gravitational potential of the CMB lensing. The intensity map is therefore possible to be used for estimating part of the lensing B-mode contributions, and subtracting it from the observed B-modes on large scales.

[78] explored expected delensing performance in future $\mathrm{CMB}$ experiments using the intensity map of the SKA radio continuum survey. Fig. 7 shows the improvement to the constraints on the tensor-to-scalar ratio of the LiteBIRD by performing the delensing analysis (for details, see [78]). We assume that the large-scale lensing B-modes of the LiteBIRD are delensed using the CMB-lensing mass fields reconstructed from the LiteBIRD and Simons Array (SA) [73], and the intensity map of the SKA radio continuum survey. The improvement is computed with varying the flux cut of the radio continuum survey. Since the future polarization sensitivity of the SA is uncertain, we show two possible cases of the SA polarization sensitivity. The inclusion of the SKA1-delensing will significantly increase the improvement to the constraints on the tensor-toscalar ratio by $80-120 \%$ compared to the case without the delensing analysis. With the SKA2 $(0.1-1 \mu \mathrm{Jy})$ and $\mathrm{CMB}$ observations, the improvement to the constraints becomes $130-160 \%$. Note that the additional improvement by the inclusion of the SKA2 to the CMB data is approximately $100 \%$. The intensity map from the SKA radio continuum survey would be quite useful for the future CMB delensing analysis, especially for the LiteBIRD observation. 


\section{Summary}

In summary, the SKA will yield transformational science aross a wide range of cosmology in the next decades. In particular, the SKA has several cosmological surveys, which have the potential to open the new frontier of cosmology and deliver the precision cosmology. The cosmology science that the SKA has the potential to provide is of great interest for SKA-Japan consortium (SKA-JP) cosmology science working group. In this paper, we have highlighted some examples of specific topics that Japanese cosmologists are currently playing an important role.

Our analysis has revealed that the SKA should possess the extremely-large survey volume, e.g. all-sky, to enhance the study of the mechanism for generating the primordial density fluctuations through the scale-dependent bias (see Sec. 2.1). In this treatment, the precise estimate of halo mass of each galaxy infered from available observables is quite essential to take the advantage of the multitracer technique. Hence, the properties of individual galaxies, such as mass and redshift, infered from available data by other surveys may provide the further improvement in the constraints.

We also expect that the SKA should explore the very high-redshift dark Universe, such as the cosmic dawn and dark age, with the 21-cm surveys (see Sec. 2.2. ). However, as already mentioned, the signals of the $21-\mathrm{cm}$ line during the EoR strongly depends on the astrophysical processes, which are poorly understood and include the large uncertainties. We should further improve the cleaning method to remove the foregrounds by working with the members of the reionization group.

Finally, we emphasize that in the SKA era the cosmological surveys will be limited by systematic errors and cosmic-variance noises rather than statistical errors, because future planned surveys will be able to probe the huge number of samples, implying that the synergy with other wavelengths is quite important and possible synergy with multiwavelength data from e.g., optical/infrared and CMB measurements, will have the potential to reduce these noises. The Japanese astronomical researchers are currently leading cosmology-oriented surveys in other wavelengths such as HSC and LiteBIRD, and participating in ongoing and upcoming experiments. By working with members of these surveys, the Japanese commity has the potential to provide the powerful tools for cosmology in next decade. The involvement of SKA-JP in the SKA will benefit our community by enlarging their expertises in cosmology science.

\section{References}

[1] P. A. R. Ade et al. [Planck Collaboration], arXiv:1502.01589 [astro-ph.CO].

[2] F. B. Abdalla et al. [Cosmology SWG Collaboration], arXiv:1501.04035 [astro-ph.CO].

[3] M. G. Santos, D. Alonso, P. Bull, M. Silva and S. Yahya, arXiv:1501.03990 [astro-ph.CO].

[4] S. Yahya, P. Bull, M. G. Santos, M. Silva, R. Maartens, P. Okouma and B. Bassett, Mon. Not. Roy. Astron. Soc. 450, no. 3, 2251 (2015) [arXiv:1412.4700 [astro-ph.CO]].

[5] M. G. Santos et al., arXiv:1501.03989 [astro-ph.CO].

[6] P. Bull, P. G. Ferreira, P. Patel and M. G. Santos, Astrophys. J. 803, no. 1, 21 (2015) [arXiv:1405.1452 [astro-ph.CO]]. 
[7] M. J. Jarvis, D. Bacon, C. Blake, M. L. Brown, S. N. Lindsay, A. Raccanelli, M. Santos and D. Schwarz, arXiv:1501.03825 [astro-ph.CO].

[8] U. Seljak, Phys. Rev. Lett. 102, 021302 (2009) [arXiv:0807.1770 [astro-ph]].

[9] N. Hamaus, U. Seljak and V. Desjacques, Phys. Rev. D 84, 083509 (2011) [arXiv:1104.2321 [astro-ph.CO]].

[10] N. Dalal, O. Dore, D. Huterer and A. Shirokov, Phys. Rev. D 77, 123514 (2008) [arXiv:0710.4560 [astro-ph]].

[11] S. Matarrese and L. Verde, Astrophys. J. 677, L77 (2008) [arXiv:0801.4826 [astro-ph]].

[12] D. Alonso, P. Bull, P. G. Ferreira, R. Maartens and M. Santos, Astrophys. J. 814, no. 2, 145 (2015) [arXiv:1505.07596 [astro-ph.CO]].

[13] S. Camera, M. G. Santos and R. Maartens, Mon. Not. Roy. Astron. Soc. 448, no. 2, 1035 (2015) [arXiv:1409.8286 [astro-ph.CO]].

[14] D. Yamauchi, K. Takahashi and M. Oguri, Phys. Rev. D 90, no. 8, 083520 (2014) [arXiv:1407.5453 [astro-ph.CO]].

[15] R. J. Wilman, L. Miller, M. J. Jarvis, T. Mauch, F. Levrier, F. B. Abdalla, S. Rawlings and H. -R. Kloeckner et al., Mon. Not. Roy. Astron. Soc. 388, 1335 (2008) [arXiv:0805.3413 [astro-ph]].

[16] S. Camera et al., arXiv:1501.03851 [astro-ph.CO].

[17] D. Alonso and P. G. Ferreira, Phys. Rev. D 92, no. 6, 063525 (2015) doi:10.1103/PhysRevD.92.063525 [arXiv:1507.03550 [astro-ph.CO]].

[18] J. Fonseca, S. Camera, M. Santos and R. Maartens, Astrophys. J. 812, no. 2, L22 (2015) doi:10.1088/2041-8205/812/2/L22 [arXiv:1507.04605 [astro-ph.CO]].

[19] T. D. Kitching, D. Bacon, M. L. Brown, P. Bull, J. D. McEwen, M. Oguri, R. Scaramella and K. Takahashi et al., arXiv:1501.03978 [astro-ph.CO].

[20] K. Takahashi et al., arXiv:1501.03859 [astro-ph.CO].

[21] T. Okamoto and W. Hu, Phys. Rev. D 66, 063008 (2002) doi:10.1103/PhysRevD.66.063008 [astro-ph/0206155].

[22] L. Boubekeur and D. H. Lyth, Phys. Rev. D 73, 021301 (2006) doi:10.1103/PhysRevD.73.021301 [astro-ph/0504046].

[23] T. Suyama and M. Yamaguchi, Phys. Rev. D 77, 023505 (2008) [arXiv:0709.2545 [astro-ph]].

[24] T. Suyama, T. Takahashi, M. Yamaguchi and S. Yokoyama, JCAP 1012, 030 (2010) [arXiv:1009.1979 [astro-ph.CO]].

[25] D. H. Lyth and D. Wands, Phys. Lett. B 524, 5 (2002) [hep-ph/0110002].

[26] T. Moroi and T. Takahashi, Phys. Lett. B 522, 215 (2001) [Erratum-ibid. B 539, 303 (2002)] [hep-ph/0110096].

[27] K. Enqvist and M. S. Sloth, Nucl. Phys. B 626, 395 (2002) [hep-ph/0109214].

[28] M. Biagetti, V. Desjacques and A. Riotto, Mon. Not. Roy. Astron. Soc. 429, 1774 (2013) doi:10.1093/mnras/sts467 [arXiv:1208.1616 [astro-ph.CO]].

[29] D. Baumann, S. Ferraro, D. Green and K. M. Smith, JCAP 1305, 001 (2013) doi:10.1088/1475-7516/2013/05/001 [arXiv:1209.2173 [astro-ph.CO]]. 
[30] K. M. Smith and M. LoVerde, JCAP 1111, 009 (2011) doi:10.1088/1475-7516/2011/11/009 [arXiv:1010.0055 [astro-ph.CO]].

[31] S. Adhikari, S. Shandera and N. Dalal, JCAP 1406, 052 (2014) doi:10.1088/1475-7516/2014/06/052 [arXiv:1402.2336 [astro-ph.CO]].

[32] D. Yamauchi and K. Takahashi, arXiv:1509.07585 [astro-ph.CO].

[33] I. Hashimoto, A. Taruya, T. Matsubara, T. Namikawa and S. Yokoyama, arXiv:1512.08352 [astro-ph.CO].

[34] T. Matsubara, Phys. Rev. D 86, 063518 (2012) [arXiv:1206.0562 [astro-ph.CO]].

[35] F. Schmidt and M. Kamionkowski, Phys. Rev. D 82, 103002 (2010) [arXiv:1008.0638 [astro-ph.CO]].

[36] L. Verde and S. Matarrese, Astrophys. J. 706, L91 (2009) [arXiv:0909.3224 [astro-ph.CO]].

[37] A. Taruya, K. Koyama and T. Matsubara, Phys. Rev. D 78, 123534 (2008) [arXiv:0808.4085 [astro-ph]].

[38] S. Yokoyama, T. Matsubara and A. Taruya, Phys. Rev. D 89, no. 4, 043524 (2014) [arXiv:1310.4925 [astro-ph.CO]].

[39] E. Sefusatti and E. Komatsu, Phys. Rev. D 76, 083004 (2007) [arXiv:0705.0343 [astro-ph]].

[40] S. Mizuno and S. Yokoyama, Phys. Rev. D 91, no. 12, 123521 (2015) [arXiv:1504.05505 [astro-ph.CO]].

[41] K. Kohri, Y. Oyama, T. Sekiguchi and T. Takahashi, JCAP 1310, 065 (2013) [arXiv:1303.1688 [astro-ph.CO]].

[42] M. Tegmark and M. Zaldarriaga, Phys. Rev. D 79, 083530 (2009) [arXiv:0805.4414 [astro-ph]].

[43] M. Tegmark and M. Zaldarriaga, Phys. Rev. D 82, 103501 (2010) [arXiv:0909.0001 [astro-ph.CO]].

[44] Y. Oyama, A. Shimizu and K. Kohri, Phys. Lett. B 718, 1186 (2013) [arXiv:1205.5223 [astro-ph.CO]].

[45] Y. Oyama, K. Kohri and M. Hazumi, arXiv:1510.03806 [astro-ph.CO].

[46] K. Kohri, Y. Oyama, T. Sekiguchi and T. Takahashi, JCAP 1409, 014 (2014) [arXiv:1404.4847 [astro-ph.CO]].

[47] M. L. Brown et al., PoS AASKA 14, 023 (2015) [arXiv:1501.03828 [astro-ph.CO]].

[48] A. Stebbins, astro-ph/9609149.

[49] M. Kamionkowski, A. Babul, C. M. Cress and A. Refregier, Mon. Not. Roy. Astron. Soc. 301, 1064 (1998) [astro-ph/9712030].

[50] D. Yamauchi, T. Namikawa and A. Taruya, JCAP 1308, 051 (2013) [arXiv:1305.3348 [astro-ph.CO]].

[51] D. Yamauchi, T. Namikawa and A. Taruya, JCAP 1210, 030 (2012) [arXiv:1205.2139 [astro-ph.CO]].

[52] S. Dodelson, E. Rozo and A. Stebbins, Phys. Rev. Lett. 91, 021301 (2003) [astro-ph/0301177].

[53] S. Saga, D. Yamauchi and K. Ichiki, Phys. Rev. D 92, no. 6, 063533 (2015) [arXiv:1505.02774 [astro-ph.CO]].

[54] D. Sarkar, P. Serra, A. Cooray, K. Ichiki and D. Baumann, Phys. Rev. D 77, 103515 (2008) [arXiv:0803.1490 [astro-ph]]. 
[55] M. Kamionkowski, A. Kosowsky and A. Stebbins, Phys. Rev. Lett. 78, 2058 (1997) [astro-ph/9609132].

[56] P. A. R. Ade et al. [BICEP2 and Keck Array Collaborations], Phys. Rev. Lett. 116, 031302 (2016) doi:10.1103/PhysRevLett.116.031302 [arXiv:1510.09217 [astro-ph.CO]].

[57] P. A. R. Ade et al. [BICEP2 and Planck Collaborations], Phys. Rev. Lett. 114, 101301 (2015) [arXiv:1502.00612 [astro-ph.CO]].

[58] P. A. R. Ade et al. [BICEP2 and Keck Array Collaborations], Astrophys. J. 811, no. 2, 126 (2015) [arXiv:1502.00643 [astro-ph.CO]].

[59] J. Dunkley et al., AIP Conf. Proc. 1141, 222 (2009) [arXiv:0811.3915 [astro-ph]].

[60] M. Betoule, E. Pierpaoli, J. Delabrouille, M. L. Jeune and J. F. Cardoso, Astron. Astrophys. 503, 691 (2009) [arXiv:0901.1056 [astro-ph.CO]].

[61] N. Katayama and E. Komatsu, Astrophys. J. 737, 78 (2011) [arXiv:1101.5210 [astro-ph.CO]].

[62] K. Ichiki, PTEP 2014, no. 6, 06B109 (2014).

[63] M. Zaldarriaga and U. Seljak, Phys. Rev. D 58, 023003 (1998) [astro-ph/9803150].

[64] U. Seljak and C. M. Hirata, Phys. Rev. D 69, 043005 (2004) [astro-ph/0310163].

[65] K. M. Smith, D. Hanson, M. LoVerde, C. M. Hirata and O. Zahn, JCAP 1206, 014 (2012) [arXiv:1010.0048 [astro-ph.CO]].

[66] T. Namikawa and R. Nagata, JCAP 1510, no. 10, 004 (2015) [arXiv:1506.09209 [astro-ph.CO]].

[67] T. Matsumura et al., Journal of Low Temperature Physics September 2014, Volume 176, Issue 5-6, pp 733-740 [arXiv:1311.2847 [astro-ph.IM]].

[68] P. A. R. Ade et al. [POLARBEAR Collaboration], Phys. Rev. Lett. 113, 021301 (2014) [arXiv:1312.6646 [astro-ph.CO]].

[69] A. van Engelen et al. [ACT Collaboration], Astrophys. J. 808, no. 1, 7 (2015) [arXiv:1412.0626 [astro-ph.CO]].

[70] K. T. Story et al. [SPT Collaboration], Astrophys. J. 810, no. 1, 50 (2015) [arXiv:1412.4760 [astro-ph.CO]].

[71] P. A. R. Ade et al. [Planck Collaboration], arXiv:1502.01591 [astro-ph.CO].

[72] T. Namikawa, PTEP 2014, no. 6, 06 B108 (2014) [arXiv:1403.3569 [astro-ph.CO]].

[73] Arnold, K., Stebor, N., Ade, P. A. R., et al. 2014, Proc. SPIE, 9153, 91531F

[74] D. Hanson et al. [SPTpol Collaboration], Phys. Rev. Lett. 111, no. 14, 141301 (2013) [arXiv:1307.5830 [astro-ph.CO]].

[75] L. Marian and G. M. Bernstein, Phys. Rev. D 76, 123009 (2007) [arXiv:0710.2538 [astro-ph]].

[76] G. Simard, D. Hanson and G. Holder, Astrophys. J. 807, 166 (2015) [arXiv:1410.0691 [astro-ph.CO]].

[77] B. D. Sherwin and M. Schmittfull, Phys. Rev. D 92, no. 4, 043005 (2015) [arXiv:1502.05356 [astro-ph.CO]].

[78] T. Namikawa, D. Yamauchi, B. Sherwin and R. Nagata, Phys. Rev. D 93, no. 4, 043527 (2016) [arXiv:1511.04653 [astro-ph.CO]]. 